\title{
Expression of Bacillus subtilis proBA Genes and Reduction of Feedback Inhibition of Proline Synthesis Increases Proline Production and Confers Osmotolerance in Transgenic Arabidopsis
}

\author{
Mingqing Chen, Hongbo Wei, JunWei Cao*, Ruijie Liu, Youliang Wang and Congyi Zheng \\ State Key Laboratory of Virology, College of Life Sciences, Wuhan University, Wuhan 430072, P. R. China \\ Received 13 November 2006, Accepted 2 January 2007
}

\begin{abstract}
Proline accumulation has been shown to correlate with tolerance to drought and salt stresses in plants. We attempt to introduce the wild-type, mutant, and fusion $\operatorname{proB} A$ genes derived from Bacillus subtilis into Arabidopsis thaliana under the control of a strong promoter cauliflower mosaic virus $35 \mathrm{~S}$ (CaMV35S). The transgenic plants produced higher level of free proline than control and the overproduction of proline resulted in the increased tolerance to osmotic stress in transgenic plants. Besides, the mutation in $\operatorname{proB} A$ genes, which were proved to lead $\gamma$-glutamyl kinase $(\gamma$-GK) reduces sensitivity to the end-product inhibition and the fusion of $\operatorname{proB}$ and $\operatorname{pro} A$ also result in increasing proline production and confer osmotolerance in transgenic lines.
\end{abstract}

Keywords: Proline, proBA gene, Transgenic Arabidopsis, Salt stress, Feedback inhibition

\section{Introduction}

The accumulation of osmolyte compounds has long been proposed as an adaptative mechanism for drought and salt tolerance, it has received increasing interest during the last 20 years. In response to drought and/or high salinity stresses, which disturb the intracellular water balance, many plants and bacteria synthesize and accumulate several kinds of compatible osmolytes, such as proline (Kavi Kishor et al., 1995), mannitol (Tarczynski et al., 1993; Shen et al., 1997), glycinebetaine (Nomura et al., 1995; Huang et al., 2000), trehalose (Garg et al., 2002), fructan (Pilon-Smits et al., 1995)

\footnotetext{
Abbreviations: $\gamma$-GK, $\gamma$-glutamyl kinase; P5CS, $\Delta 1$-pyrroline-5-carboxilate synthetase; CaMV35S ,cauliflower mosaic virus $35 \mathrm{~S}$.
}

\footnotetext{
* To whom correspondence should be addressed.

Tel: 86-027-62772461; Fax: 86-027-68752560

E-mail: caojw6131@126.com
}

that function as osmotica. Among the compatible solutes, proline appears to be the most widely distributed osmolyte accumulated under stress conditions not only in plants but also in eubacteria.

The pathway of proline synthesis from glutamate, the most common mechanism of proline biosynthesis, comprises three enzymatic steps (Fig. 1). The corresponding genes of Bacillus subtilis, proB, pro $A$, and proC, encode $\gamma$-glutamyl kinase $(\gamma$ GK), $\gamma$-glutamyl-phosphate reductase ( $\gamma$-GPR) and $\Delta$-pyrroline5-carboxylate (P5C) reductase. The proline pathway in plants has been proposed to be analogous to that in bacteria, although proline can also be made from ornithine (Adams and Frank, 1980). In plants, the $\Delta 1$-pyrroline-5-carboxilate synthetase (P5CS) is a bifunctional enzyme with both $\gamma$-GK and glutamate semialdehyde (GSA) dehydrogenase activities and it is a rate-limiting enzyme for biosynthetic pathway in higher plants, being feedback inhibited by proline (Hu et al., 1992; Zhang et al., 1995). In bacteria, proline biosynthesis has been shown to be regulated by the end-product inhibition of $\gamma$ glutamyl kinase ( $\gamma$-GK) activity (Smith et al., 1984). A similar situation was also observed in plants. The $\gamma$-GK activity of Vigna P5CS was inhibitive by proline, but its GSA dehydrogenase activity was not affected, suggesting that the $\gamma$ GK is the rate-limiting step in proline biosynthesis in plants (Zhang et al., 1995).

In recent years, several studies have demonstrated that genetic manipulation of the accumulation of compatible solutes resulted in increased tolerance to drought or salt stress in transgenic plants (Tarczynski et al., 1993; Kavi Kishor et al., 1995; Nomura et al., 1995; Pilon-Smits et al., 1995; Shen et al., 1997; Huang et al., 2000; Garg et al., 2002). Since proline biosynthesis during stress has been demonstrated to be controlled by P5CS activity, many attempts to enhanced osmotolerance by increasing proline accumulation centered on this enzyme. The $\Delta 1$-pyrroline-5-carboxylate synthetase (P5CS) cDNA from mothbean (Vigna aconitifolia L.) was introduced into rice (Oryza sati6a L.) (Shen et al., 1998) and tobacco (Kavi Kishor et al., 1995). 




Fig. 1. Pathways of proline biosynthesis from glutamate in $B$. subtilis and plants. Steps in pathway are the same in both bacteria and plants, but the initial two reactions are performed by the bifunctional P5CS enzyme in plants.

However, it has not been reported that introduced proBA genes into plants. It was reported that the monthbean P5CS cDNA clones complemented Escherichia coli proB, proA and proBA mutants deficient in $\gamma$-GK, GSA dehydrogenase and both enzymes, respectively (Hu et al., 1992); however, it is not known whether $\gamma$-GK and GSA dehydrogenase exhibited enzymatic activity as P5CS in plants.

Since proline biosynthesis of $\gamma$-GK or P5CS is subject to feedback inhibition by the elevated level of proline, some work attempt to reduce the sensitivity of these enzymes to end-product inhibition. It was extensively studied that mutations of $p r o B$ gene led $\gamma-\mathrm{GK}$ reduce sensitivity to proline -mediated feedback inhibition, resulting that proline overproduced and osmotolerance enhanced in bacteria (Csonka, 1981; Dandekar and Uratsu, 1988; Sleator et al., 2001). A similar situation was also observed in Vigna P5CS, the substitution of an alanine for a phenylalanine at amino acid residue 129 of the $\mathrm{P} 5 \mathrm{CS}$ resulted in a significant reduction of proline feedback inhibition (Zhang et al., 1995). The mutation responsible for the resistance of proB74 encoded mutant $\gamma$-GK to proline inhibition involves a substitution of an $A$ to a $G$ nucleotide with a consequent alteration of aspartate at position 107 in the polypeptide to asparagines (Csonka et al., 1988; Dandekar and Uratsu, 1988). This aspartate residue (at position 128) conserved in the Vigna aconitifolia P5CS is not involved in the feedback inhibition (Zhang et al., 1995), while a replacement of Phe at position 129 by Ala resulted in removal of feedback inhibition of P5CS (Zhang et al., 1995; Hong et al., 2000).

In our previous work, B. subtilis 93151 was treated with Nmethy-N'-nitro-N-nitrosoguanidine (NTG). Subsequently, we obtained a mutant (B. subtilis 93151-14) which could resist high $\mathrm{NaCl}(14 \%)$ stress by screening in minimal medium (MM) containing 3, 4-didehydroproline. The proBA genes were cloned from the wild strain and mutant, respectively. We found mutation sites in proB, which lead in an amino acid substitution (Ser216Thr), also in proA genes, which resulted three residue changes $(\mathrm{Thr} 150 \rightarrow$ Ala, Arg408 $\rightarrow$ Lys, Gln412 $\rightarrow$ Arg) (Miao et al., 2002; Liu et al., 2004). Besides, the $p r o B$ and proA genes from B.subtilis overlap by $4 \mathrm{nt}$ and this leads to the formation of a tight genic domain (GenBank accession number AY601668) (Miao et al., 2002; Liu et al., 2004). A fusion proBA gene was constructed by removing the stop codon of proB gene and replacing it with a restriction enzyme site to serve as a linker between the mutated proB and pro $A$ genes, which expressed an artificial bifunctional enzyme, $\gamma$-glutamyl kinase $/ \gamma$-glutamyl phosphate reductase $(\gamma$ GK/ $\gamma$-GPR) (Liu et al., 2005).

In this work, we attempt to introduce the wild-type, mutant, and fusion proBA genes derived from $B$. subtilis into Arabidopsis thaliana Another goal for this research was to establish the expression of proBA in Arabidopsis.

\section{Materials and Methods}

Plant material and growth conditions. Sterilized seeds of Arabidopsis ecotype Columbia (Col-0) were used for all transformation experiments and subsequent tissue analysis. Plants were either grown in vermiculite in an environmentally controlled green house at $24^{\circ} \mathrm{C}$ with $16 / 8 \mathrm{~h}$ of light/dark cycle and $80 \%$ humidity or aseptically on germination in medium (half strength Murashige-Skoog salts pH 5.7 with $\mathrm{KOH}, 0.8 \%$ agar) under continuous light at $24^{\circ} \mathrm{C}$.

Isolation of B. subtilis proBA genes and plasmid construction. The wild-type and mutant proBA genes were cloned from the wildtype strain B. subtilis 93151 and mutant strain B. subtilis 93151-14, respectively (Miao et al., 2002). The fusion proBA gene was constructed by removing the stop codon of $p r o B$ gene and replacing it with a restriction enzyme site to serve as a linker between the mutant proB and proA genes (Liu et al., 2005). These sequences were subcloned into $\mathrm{pBE} 2$ and the positive constructs were named as $\mathrm{pBE} 2 / p r o B A / \mathrm{W}, \mathrm{pBE} 2 / p r o B A / \mathrm{M}$ and $\mathrm{pBE} 2 / p r o B A / \mathrm{MF}$ respectively. The wild-type and mutant proBA genes were amplified by PCR from $\mathrm{pBE} 2 / \mathrm{proBA} / \mathrm{W}, \mathrm{pBE} 2 / p r o B A / \mathrm{M}$ with primers $\mathrm{P} 1$ (5'ggctctagaacggagg agaaactat gaaa-3', the underlining indicates the positions of XbaI) and P2 (5'-ggcgagctcatcgtcaatctcccegcaca-3', the underlining indicates the positions of $S a c I)$. The proBA fusion gene were amplified by PCR from $\mathrm{pBE} 2 /$ proBA/MF with primers P3 (5'ggcggatccgaaactatgaaaaag- 3 ', the underlining indicates the positions of BamHI) and P2. The wild-type and mutant proBA genes were ligated into the $\mathrm{Xba \textrm {I }} / \mathrm{SacI}$ sites and the fusion proBA gene were ligated into the $\mathrm{BamHI} / \mathrm{SacI}$ sites of the binary vector $\mathrm{pMD} 1$ which was promoted by the cauliflower mosaic virus 35S (CaMV35S) promoter and terminated by nopaline synthase terminator. The constructs were transformed into E. coli $\mathrm{DH} 5 \alpha$ and the positive colonies were further characterized by the restriction enzyme analysis and PCR analysis. The resulting recombinant plasmids named pMD1-proBA/W, pMD1-proBA/M and pMD1-proBA/MF, respectively. These recombinant plasmids and the control plasmid 
pMD1 were transformed into Agrobacterium tumefaciens strain LBA4404. The positive colonies were characterized by PCR analysis.

Plant transformation. Arabidopsis plants were transformed using a modified vacuum infiltration method (Bechtold et al., 1993). Arabidopsis seeds produced by the first generation of transformed plants were harvested and selected on half strength Murashige-Skoog salts medium containing $50 \mathrm{mg} / \mathrm{l}$ kanamycin. The 10-day-old seedlings can be different on the $1 / 2 \mathrm{MS}$ medium containing $50 \mathrm{mg} / \mathrm{l}$ kanamycin. The resulting kanamycin-resistant plants (T1) were transferred to soil in pots and grown in chambers at 24 with $16 / 8 \mathrm{~h}$ of light/dark cycle and $80 \%$ humidity for the production of seeds (T2).

PCR analysis of transgenic plants. Transgenic Arabidopsis seeds (T2) were germinated on MS medium containing Kanamycium for ten days then transferred to soil in pots. The total DNA was extracted from the leaves of transgenic Arabidopsis seedlings of T2 generation. Leaf extracts were analyzed by PCR for the presence of the proBA genes or proBA fusion gene using the primer $\mathrm{P} 1$ and $\mathrm{P} 2$. The positive transgenic lines were grown for the production of seeds (T3).

Northern-blot analyses. Total RNA isolated from the transgenic and control Arabidopsis seedlings was electrophoresed, blotted, and hybridized with the DNA fragment corresponding to the $p r o B$ gene as a radioactive probe. Hybridization and washing of the filters were preformed by the method of Sambrook's (Sambrook et al., 1989).

Germination under salt stress. $200 \mu \mathrm{l}$ sterilized seeds of wild type and T3 transgenic Arabidopsis were cultured in MS medium with 0 and $300 \mathrm{mM} \mathrm{NaCl}$ on a glass plate for 10 days after $48 \mathrm{~h}$ at $4^{\circ} \mathrm{C}$.

Salt treatment on Arabidopsis. The seeds of wild type and T3 transgenic Arabidopsis were germinated in vermiculite in small pots with holes in the bottom (4 plants per pot). The pots were kept in flat-bottomed trays. The seedlings were grown for 4-5 weeks. For salt stress, 4-week-old plants were watered with equal volume Hoagland solution containing $200 \mathrm{mM} \mathrm{NaCl}$ for 20 days, and the $\mathrm{NaCl}$ solution was added every 2 days to maintain the constant concentration of $\mathrm{NaCl}$ in the vermiculite. The stressed plants were re-supplied with water to allow them recover and grow without stress.

Measurement of proline contents. Leaves of wild type and T3 transgenic Arabidopsis were used for determination of proline content as described previously (Bates et al, 1973). Precaution was taken to select leaves of similar age and size. About $0.5 \mathrm{~g}$ of leaf tissue collected from normal and stressed leaves was extracted overnight in $1 \mathrm{ml} \mathrm{3 \%}(\mathrm{w} / \mathrm{v})$ aqueous 5-sulphosalicylic acid. Precipitated protein and other debris were removed by centrifugation at $8,000 \mathrm{~g}$ for $10 \mathrm{~min}$. Acid ninhydrin was prepared by dissolving $1.25 \mathrm{~g}$ ninhydrin in $30 \mathrm{ml}$ glacial acetic acid and $20 \mathrm{ml} 6 \mathrm{M} \mathrm{H}_{3} \mathrm{PO}_{4}$. The cellular extract was mixed with $0.5 \mathrm{ml}$ acid ninhydrin and $0.5 \mathrm{ml}$ glacial acetic acid. The mixture reacted in $100^{\circ} \mathrm{C}$ for $1 \mathrm{~h}$. Samples were cooled and mixed vigorously with $2 \mathrm{ml}$ toluene. The absorbance of samples was read at $520 \mathrm{~nm}$ against a toluene blank.
Values of proline contents were taken from measurements of three independent samples.

Extract preparation and enzyme assay. $0.5 \mathrm{~L}$ bacterial cultures of LB broth containing $80 \mu \mathrm{g} \mathrm{ml} l^{-1}$ of ampicillin were grown at $37^{\circ} \mathrm{C}$ with shaking until mid-log phase. Cultures were harvested by centrifugation, washed in TD buffer $(50 \mathrm{mM}$ Tris, $1 \mathrm{mM}$ dithiothreitol, $\mathrm{pH}$ 7.2). Cell-free extracts were prepared by resuspending cell pellets in TD buffer followed by sonication. Cellular debris was removed by centrifugation at $14,000 \mathrm{~g}$ for $20 \mathrm{~min}$ at $4^{\circ} \mathrm{C}$. The activities of GK and GPR in crude bacterial homogenates were determined using the coupled reaction (Smith et al., 1984). The buffer contained $100 \mathrm{mM}$ Tris- $\mathrm{HCl} \mathrm{pH}$ 7.2, $50 \mathrm{mM}$ glutamate, 0.15 $\mathrm{mM}$ NAPDH, $1 \mathrm{mM} \mathrm{MgCl}_{2}, 5 \mathrm{mM}$ ATP and $1 \mathrm{mM}$ DTT. The oxidation of NAPDH was monitored at $340 \mathrm{~nm}$. One unit of GK/ GPR corresponds to the oxidation of 1 nmole NAPDH per minute at room temperature. Protein concentrations were determined according to Bradford using bovine serum albumin as standard (Bradford, 1976).

\section{Results}

Production of transgenic plants. The genes derived from $B$. subtilis: the wild-type proBA genes, the mutant pro $B A$ genes, and the fusion proBA gene respectively were transformed into Arabidopsis using a modified vacuum infiltration method. Selected on half strength MS salts with $50 \mathrm{mg} / \mathrm{l}$ kanamycin, about $0.2 \%$ seedlings of the total were proved antibioticresistant lines. To confirm their transgenic nature, PCR amplifications were performed. Among the antibiotic-resistant lines, 23 lines carrying the wild-type proBA genes, 27 lines carrying the mutant proBA genes and 49 lines carrying the fusion proBA gene were PCR+ (Fig. 2). The transgenic lines carrying the wild-type proBA genes, the mutant proBA genes and the fusion $p r o B A$ gene respectively named as $A t p r o B A / \mathrm{w}$, AtproBA/M and AtproBA/MF, the control transgenic lines carrying the empty vector pMD1 named AtpMD1.

Northern blot analyses of transgenic Arabidopsis. The proBA genes transcriptional levels of transgenic Arabidopsis



Fig. 2. PCR analysis confirming the presence of gene into Arabidopsis genome Lane 1: $\lambda \mathrm{DNA} /$ HindIII marker; Lane 2, wild-type Arabidopsis plant; Lanes3, 4: transformants carry the wild-type proBA genes of $B$. subtilis; Lanes 5, 6: transformants carry the mutant proBA genes of $B$. subtilis; Lanes 7: transformants carry the fusion proBA gene of B. subtilis; Lane 8: pMD1 plant. 


\section{proBA Transgenic \\ WT pMD1 proBA/W proBA/M proBA/MF}

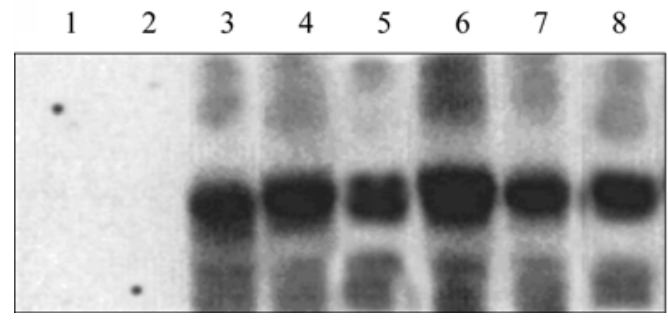

Fig. 3. Expression of proBA genes in transgenic Arabidopsis plants. Northern blot of total RNA from wild-type (WT) and pMD1 plants (lanes 1 and 2) and the proBA transgenic lines: AtproBA/w (Lanes 3-4), AtproBA/M (Lanes 5-6) and AtproBA/ MF (Lanes 7-8).

lines were analyzed by the Northern blot. The results showed that the proBA genes constitutively expressed as expected in transgenic Arabidopsis (Fig. 3).

Germination under salt stress. We examined the effects of salt stress on the germination of seeds from the wild type and the proBA-transgenic plants (Fig. 4). After five days, $100 \%$ germination was observed in the seeds from wild-type as well as transgenic plants in the presence of $50 \mathrm{mM} \mathrm{NaCl}$ or in its absence (Fig. 4A). At $200 \mathrm{mM} \mathrm{NaCl}$, more than $50 \%$ of the transformed plants seeds germinated, whereas only $30 \%$ of the seeds of the wild-type plant did so. At $250 \mathrm{mM} \mathrm{NaCl}$, seeds of the wild-type plant did not germinate at all, whereas $10 \%$ of seeds of AtproBA/w, 30\% of AtproBA/M and $40 \%$ of AtproBAMF transgenic plants germinated. These findings indicate that the transformed plants had enhanced ability to germinate under salt-stress conditions.

In the proBA-transgenic plants, the transgenic lines carrying the mutant $p r o B A$ genes germinated faster than the wild-type proBA-transgenic lines in $100-300 \mathrm{mmol} \mathrm{NaCl}$ (Fig. 4B). At $300 \mathrm{mM} \mathrm{NaCl}$, some of AtproBA/M and AtproBA/MF transgenic plants germinated whereas no AtproBA/w transgenic lines did so. It seems that the mutation in the proBA genes confer transgenic plants tolerance to salt-stress in some extent.

The fusion proBA-transgenic lines showed the best ability to germinate under salt-stress: its percent of germination was the highest in all kinds of $\mathrm{NaCl}$ contents (Fig. 4).

Growth under salt stress. Fig. 5 shows the effects of salt stress on the growth of seedlings after germination. Seeds of the wildtype plant and the T3 seeds of the fusion proBA-transgenic plants were allowed to germinate on solidified Murashige and Skoog's medium supplemented with $100 \mathrm{mM} \mathrm{NaCl}$, and roots and cotyledons were allowed to develop for a further 20 days. The growth of the wild-type plants was weak and the leaves turned white after 10 days. By contrast, the transformed plants remained good growth. One hundred lines of AtproBA/MF transgenic plants and the wild-type plants were tested to assess


Fig. 4. The effects of salt stress on the germination of seedlings wild-type and transformed plant. Seeds from wild-type plants and T3 seeds from proBA-transformed plants were germinated on plates of $1 / 2 \mathrm{MS}$ medium that had been supplemented with $\mathrm{NaCl}$ (from 0 to $350 \mathrm{mM}$ ) after incubation at $4^{\circ} \mathrm{C}$ for 2 days. Results are means \pm S.E. (A) The percent of germination of wild-type and transformed plants after five days under several of $\mathrm{NaCl}$ content. (B) The effects of germination of seedlings wildtype and transformed plant under $150 \mathrm{mM} \mathrm{NaCl}$-stress.

the development of the roots. The development of the roots of the transformed plants was markedly more efficient than that of the wild-type plants. The root length of AtproBA/MF transgenic plants was 2 to 3-fold longer than that of the wild-type plants (Fig. 5B). Uniform growth rates for all of wild type and transformed plants were observed in the absence of $\mathrm{NaCl}$. The rates of growth in the presence of $\mathrm{NaCl}$ were lower than those in the absence of $\mathrm{NaCl}$. At $200 \mathrm{mM} \mathrm{NaCl}$, the transformed plants grew slowly, whereas the wild-type plants did not grow at all. At $250 \mathrm{mM} \mathrm{NaCl}$, no growth was observed in both transformed and wild-type plants.

Tolerance of mature plants to salt stress. To determine the tolerance of mature plants to salt stress, we measured the dry 

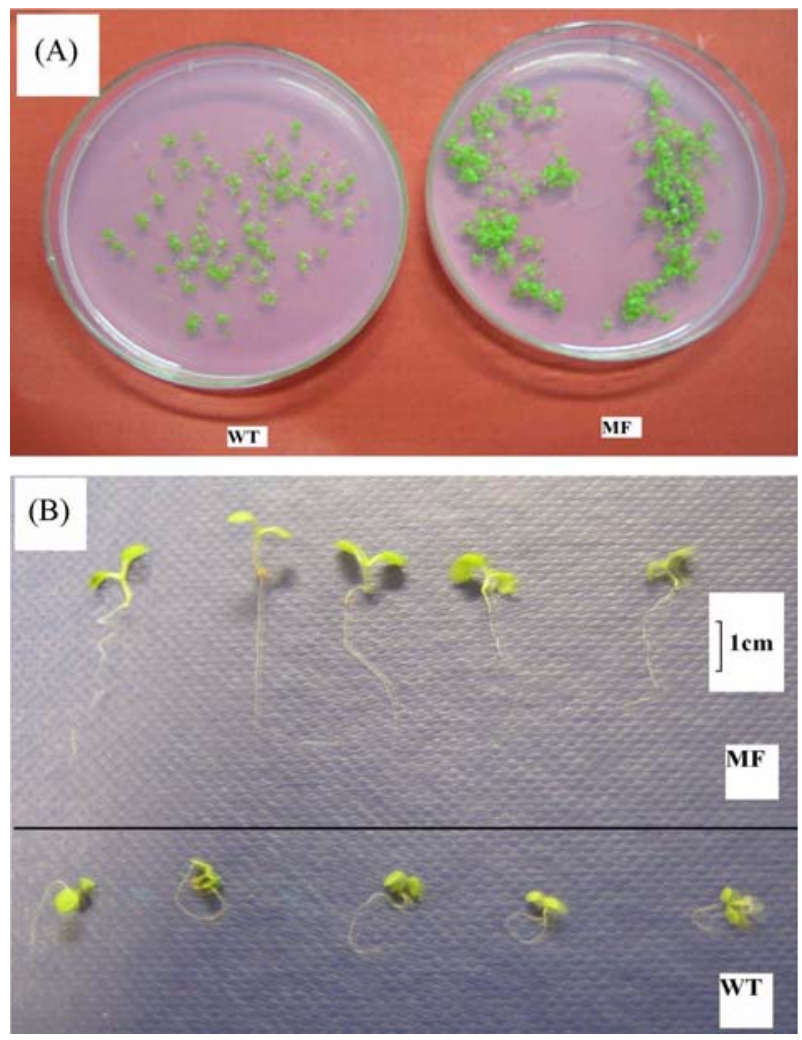

Fig. 5. Effects of salt stress on the growth after germination of wild-type and transformed plant. Seeds from wild-type plants and T3 seeds from AtproBA/MF transformed plants were germinated on plates of MS medium that had been supplemented with $100 \mathrm{mM} \mathrm{NaCl}$. After incubation at $4^{\circ} \mathrm{C}$ for 2 days, the plates were kept in the vertical position at $24^{\circ} \mathrm{C}$ for 6 days under continuous light. (A) Effects of the growth on the plates (B) Effects of the development of the roots.

weight of control and transgenic plants under normal and 200 $\mathrm{mM} \mathrm{NaCl}$ stressed conditions. Control and transgenic plants did not differ when grown under normal conditions (Fig. 6A). However, after 10 days streess, the control plants (the wild-type Arabidopsis plants and pMD1 transgenic plants) and the wildtype proBA-transgenic plants started to wilt and the leaves began to turn yellow, whereas the mutant proBA-transgenic plants and the fusion proBA-transgenic plants still showed healthy growth. After 20 days of $\mathrm{NaCl}$ stress, the control plants died away, whereas the mutant proBA-transgenic plants and the fusion proBA-transgenic plants started to seed (Fig. 6B). At last, $80 \%$ of 50 lines AtproBA/M and $90 \%$ of 70 lines AtproBA/MF could adapt and complete their life cycle under $200 \mathrm{mM} \mathrm{NaCl}$ stress. Compared to control plants, AtproBA/M plants had 1.9fold greater biomass and AtproBA/MF plants had 2.2-fold greater biomass (Fig. 6A). Significant difference between control and proBA-transgenic plants (the mutant and the fusion) in growth under salt-stressed conditions demonstrated introduction the mutant proBA genes in plants may lead to increased tolerance to drought and salinity stress.

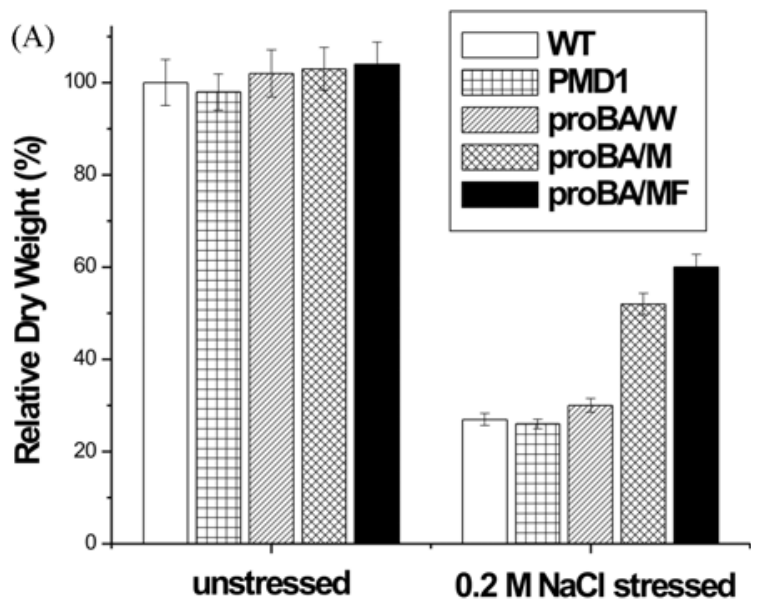

(B)

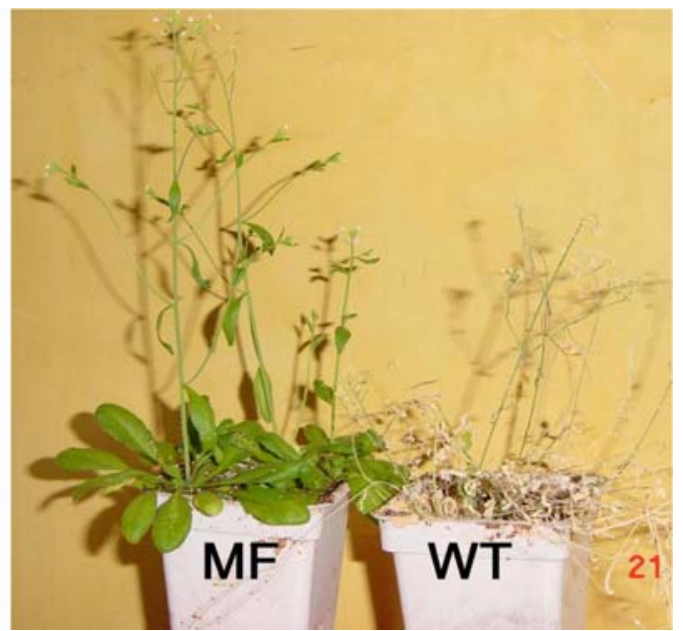

Fig. 6. Effects of salt stress on mature plants of wild-type and the fusion proBA-transgenic plants. 4-week-old plants were watered with equal volume Hoagland solution containing $200 \mathrm{mM} \mathrm{NaCl}$ for 20 days, and the $\mathrm{NaCl}$ solution was added every 2 days to maintain the constant concentration of $\mathrm{NaCl}$ in the vermiculite. (A) Comparison of the wild-type, pMD1, and proBA-transgenic plants at normal and $0.2 \mathrm{M} \mathrm{NaCl}$ salt-stressed conditions. 30 seedlings were dried and weighed. Data are means \pm S.D. (B) Growth performance of wild-type and the fusion proBA-transgenic plants at normal and $0.2 \mathrm{M} \mathrm{NaCl}$ salt-stressed conditions.

Free proline level in transgenic plants. Proline content in the wild-type Arabidopsis and the transgenic lines including AtpMD1, AtproBA/w, AtproBA/M and AtproBA/MF respectively was determined at normal and salt-stressed conditions. We measured the free proline contents of new leaf cells of 5week-old plants. Proline levels were increased in both control and transgenic plants when it was imposed $200 \mathrm{mM} \mathrm{NaCl}$ stress (Fig. 7). This values increased from $185 \mu \mathrm{g} \mathrm{g}^{-1}$ fresh weight (before stress) to about $3000 \mu \mathrm{g} \mathrm{g}^{-1}$ fresh weight (after stress) in control (wild-type and pMD1) plants, from $315 \mu \mathrm{g}$ $\mathrm{g}^{-1}$ to about $3423 \mu \mathrm{g} \mathrm{g}^{-1}$ in the wild-type proBA-transgenic lines, from $315 \mu \mathrm{g} \mathrm{g}^{-1}$ to about $5876 \mu \mathrm{g} \mathrm{g}^{-1}$ in the mutant proBA-transgenic lines, and from about $300 \mu \mathrm{g} \mathrm{g}^{-1}$ to an 




Fig. 7. Proline content in leaves of the wild-type and pMD1, AtproBA/w, AtproBA/M and AtproBA/MF transgenic plants at normal and $0.2 \mathrm{M} \mathrm{NaCl}$ salt-stressed conditions. Data are mean and S.E.

average of $6543 \mu \mathrm{g} \mathrm{g}^{-1}$ in the fusion proBA-transgenic lines.

Without stress, proline content was approximately 2- fold greater in transgenic lines than in control plants. After stress, no significant differences in proline content were observed in the wild-type proBA-transgenic lines when compared with the control (wild-type and pMD1) plants. However, the level of free proline was 2 to 2.5 -fold greater in the mutant proBAtransgenic lines and the fusion proBA-transgenic lines than in control plants after stress. Proline content in the fusion proBAtransgenic lines was greater than in the mutant proBAtransgenic lines after $200 \mathrm{mM} \mathrm{NaCl}$ stress.

Effect of proBA mutation and fusion on the activity of GK/ GPR. To assess the effect of proBA mutation and fusion on the activity of GK/GPR, the wild-type, mutant, and fusion proBA gene respectively were ligated into vector $\mathrm{pBE} 2$ and transformed into E.coli JM83 ( $\triangle p r o B A)$ (Fiedler and Skerra 2001). Cell homogenates of above transformants were analyzed for the activities and the response to feedback inhibition of GK/GPR. The wild-type GK/GPR produced by the plasmid $\mathrm{pBE} 2 /$ proBA-W is very sensitive to prolinemediated feedback inhibition (Fig. 8). However, the mutant GK/GPR was about 30-fold less sensitive to proline inhibition than the wild-type enzyme. Fifty percent inhibition of the wild-type enzyme was observed in the presence of $7.5 \times 10^{-6}$ $\mathrm{M}$ proline, whereas $2.5 \times 10^{-4} \mathrm{M}$ proline was required to inhibit $50 \%$ of the mutant enzyme activity. Furthermore, the fused GK/GPR was about 2-fold more active than the separate GK and GPR.

\section{Discussion}

There are conflicting evidence about proline role and the relevance of its accumulation in the maintenance of yield. In


Fig. 8. Effect of proBA mutation and fusion on the specific activity of GK/GPR in the presence or absence of L-proline. GK/GPR activities of JM83/ proBA-W, JM83/ proBA-M, JM83/ proBA-MF were measured by the stand coupled assay. Samples assayed in the absence and presence of L-proline as indicated, respectively. Data are means of three independent determinations. (A) Effect of the proBA mutation and fusion on the specific activity of GK/GPR in the presence or absence of L-proline. Results are means \pm S.E. (B) Effect of L-proline on the relative activity of GK/GPR.

many report, osmolyte accumulation has been proposed as an effective tolerance mechanism for water deficits and salt tolerance (Tarczynski et al., 1993; Nomura et al., 1995; PilonSmits et al., 1995; Shen et al., 1997; Huang et al., 2000; Garg et al., 2002), which could be enhanced in crops (Kavi Kishor et al., 1995). Others have suggested that osmolyte accumulation and crop yield have no consistent benefit, with probably no link with osmotic adjustment (Blum et al., 1996; Hare et al., 1998; Serraj and Sinclair, 2002). In this work, the development of the roots of the transformed plants was markedly more efficient than that of the wild-type plants in the presence of $100 \mathrm{mM} \mathrm{NaCl}$ (Fig. 5B). The mutant proBA-transgenic plants and the fusion proBA-transgenic plants could adapt and complete their life cycle under $200 \mathrm{mM} \mathrm{NaCl}$ stress. These findings suggested that proline accumulation have positive effect on osmotolerance and root length under salt stress. The 
transgenic plants produced higher level of free proline than control suggested that overexpression of proBA genes leads to proline accumulation in the transgenic lines and $p r o B A$ genes derived from $B$. subtilis could perform function as P5CS in Arabidopsis.

In $B$. subtilis 93151, the proB and pro $A$ constitute an operon and are suggested to be overlapped by $4 \mathrm{nt}$. When the wildtype and mutant proBA genes were introduced into Arabidopsis thaliana, the transcripts of proBA genes might be readthrough and express a fusion protein as P5CS in transgenic plants AtproBA/w and AtproBA/M, because the expression system of plants could not recognize the promoter and SD sequence ahead of proA gene.

Proline biosynthesis in many bacteria is frequently regulated through feedback inhibition of the first biosynthetic enzyme $(\gamma-\mathrm{GK})$. The intracellular concentration of proline is raised to over $1 \mathrm{M}$ under truly hypertonic growth conditions (Kempf and Bremer, 1998). Scince the $\gamma$-GK of Gramnegative organisms is inhibited by relatively low proline concentrations, it was thought that the regulation of salt tolerant Gram-positive bacteria should be somewhat different in proline-producing (Whatmore et al., 1990). In this work, proline was found to inhibition both the mutant and wild-type $\gamma$-GK/ $\gamma$-GPR, but a 30 -fold greater concentration of proline was required to inhibition the mutant enzyme than was required to inhibit the wild-type enzyme. Thus we presumed that $\gamma$-GK enzyme in $B$. subtilis also is subject to feedback inhibition by proline. In addition to proBA pathway, recent studies revealed an additional proline biosynthetic pathway: proHJ, which is responsible for the high-level accumulation of proline under hyper-osmotic growth conditions (Wood et al., 2001).

For proBA-transgenic plants, reduction of feedback inhibition in the mutant proBA-transgenic lines resulted in a 2-fold increase in proline content compared with that in the wildtype proBA-transgenic lines. This difference also show that feedback regulation of the wild-type $\gamma$-GK in proBA-transgenic lines is not completely eliminated under stress, because the wild-type proBA-transgenic lines and the mutant proBAtransgenic lines should accumulate uniform proline content if the wild-type $\gamma$-GK completely lose its feedback regulation property.

To obtain an expected $\gamma$-GK $/ \gamma$-GPR enzyme complex that may function as bifunctional enzyme (P5CS), the fusion proBA gene was constructed by fusing the mutated $p r o B$ and pro $A$ genes. The fused GK/GPR was about 2-fold more active than the separate GK and GPR.

In this test, expression of the fused proBA rendered transgenic plant accumulation more proline at $200 \mathrm{mM} \mathrm{NaCl}$ stress and more salt-tolerance than expression of the separate mutant proBA. Thus, in addition to the mutation of proB confers the transformants less sensitivity to the end -product inhibition, the fusion of proB and proA also contributed to the overproduction of proline. One plausible explanation for the increased proline content is that the fusion proBA gene should be more propitious to express a fusion protein than the wildtype proBA in transgenic plants. The artificial bifunctional enzyme was propitious to ensure closeness between the enzyme moieties and increase substrate transfer taken place between GK and GRP. Such proximity reduces the breakdown of the labile $\gamma$-glutamyl-phosphate which thereby increases the overall proline production.

Acknowledgments This work was supported by the Innovation Fund in Wuhan University of P. R. China.

\section{References}

Adams, E. and Frank, L. (1980) Metabolism of proline and the hydroxyprolines. Annu. Rev. Biochem. 49, 1005-1061.

Bates, L. S., Waldren, R. P. and Teeare, I. D. (1973) Rapid determination of free proline for water-stress studies. Plant Soil 39, 205-207.

Bechtold, N., Ellis, J. and Lelletier, G. (1993) In planta Agrobacterium mediated gene transfer by infiltration of adult Arabidopsis thaliana plants. C. $R$ Acad. Sci. Paris Life Sci. 316, 1194-1199.

Blum, A. (1996) Crop responses to drought and the interpretation of adaptation. Plant Growth Regulation 20, 135-148.

Bradford, M. M. (1976) A rapid and sensitive method for the quantitation of microgram quantities of protein utilizing the principle of protein-dye binding. Anal. Biochem. 72, 248-254.

Csonka, L. N. (1981) Proline over-production results in enhanced osmotolerance in Salmonella typhimurium. Mol. Gen. Genet. 182, 82-86.

Csonka, L. N., .Gelvin, S. B, Goodner, B. W., Orser, C. S., Siemieniak, D. and Slightom, J. L. (1988) Nucleotide sequence of a mutation in the proB gene of Escherichia coli that confers proline overproduction and enhanced tolerance to osmotic stress. Gene 64, 199-205.

Dandekar, A. M. and Uratsu, S. L. (1988) A single base pair change in proline biosynthesis genes causes osmotic stress tolerance. J. Bacteriol. 170, 5943-5945.

Fiedler, M. and Skerra, A. (2001) proBA complementation of an auxotrophic $E$. coli strain improves plasmid stability and expression yield during fermented production of a recombinant antibody fragment. Gene 274, 111-118.

Garg, A. K., Kim, J. K., Owens, T. G., Ranwala, A. P., Choi, Y. D., Kochian, L. V. and Wu, R. (2002) Trehalose accumulation in rice plants confers high tolerance levels to different abiotic stresses. Proc. Natl. Acad. Sci. USA 99, 15898-15903.

Hare, P. D., Cress, W. A. and Van Staden, J. (1998) Dissecting the roles of osmolyte accumulation during stress. Plant, Cell and Environment 21, 535-553.

Hong, Z., Lakkineni, K., Zhang, H. and Verma D. P. S. (2000) Removal of feedback inhibition of D1-pyrroline-5-carboxylate synthetase results in increased proline accumulation and protection of plants from osmotic stress. Plant Physiol. 122, 1129-1136.

Hu, C. A., Delauney, A. J. and Verma, D. P. (1992) A bifunctional enzyme (D1-pyrroline-5-carboxylate synthetase) catalyses the first two steps in proline biosynthesis in plants. Proc. Natl. Acad. Sci. USA 89, 9354-9358. 
Huang, J., Hirji, R., Adam, L., Rozwadowski, K. L., Hammerlind, J. K., Keller, W. A. and Selvaraj, G. (2000) Genetic engineering of glycinebetaine production toward enhancing stress tolerance in plants: metabolic limitations. Plant Physiol. 122, 747-756.

Kavi Kishor, P. B., Hong, Z., Miao, G., Hu, C. A. and Verma, D. P. S. (1995) Overexpression of D 1-pyrroline-5-carboxylate synthetase increases proline production and confers osmotolerance in transgenic plants. Plant Physiol. 108, 1387-1394.

Kempf, B. and Bremer, E. (1998) Uptake and synthesis of compatible solutes as microbial stress responses to highosmolality environments. Arch. Microbiol. 170, 319-330.

Liu, R. J., Cao, J. W. and Miao, L. X. (2004) proA gene cloning and function analysis of proBA gene in osmoregulation from a salt-tolerant mutant of Bacillus subtilis. Acta. Microbiol. Sinica. 44, 452-456.

Liu, R. J., Chen, T. and Cao, J. W. (2005) Construction of fused osmoregulation proBA gene from a salt-tolerant mutant of Bacillus subtilis and its influence on the osmotolerance of $E$. coli. Acta Microbiol. Sinica. 45, 23-26.

Miao, L. X., Cao, J. W., Liu, R. J., Wang, Y. L. and Zeng, Y. H. (2002) Cloning and sequencing of proBA gene from the selected mutant resistant to proline analogue from Bacillus subtilis. Acta. Genetica. Sinica. 29, 1111-1117.

Nomura, M., Ishitani, M., Takabe, T., Rai, A. K. and Takabe, T. (1995) Synechococcus sp. PPC 7942 transformed with E. coli bet genes produces glycine betaine from choline and acquires resistance to salt stress. Plant Physiol. 107, 703-708.

Pilon-Smits, E. A. H., Ebskamp, M. J. M., Paul, M. J., Jeuken, M. J. W., Weisbeek, P. J. and Smeekens, S. C. M. (1995) Improved performance of transgenic fructan-accumulating tobacco under drought stress. Plant Physiol. 107, 125-130.

Sambrook, J., Fritsch, E. F. and Maniatis, T. (1989) Molecular Cloning: A Laboratory Manual, 2nd ed., Cold Spring Harbor
Laboratory Press, New York, USA.

Serraj, R. and Sinclair, T. R. (2002) Osmolyte accumulation: can it really help increase crop yield under drought conditions? Plant, Cell and Environment 25, 333-341

Shen, B., Jensen, R. G. and Bohnert, H. J. (1997) Increased resistance to oxidative stress in transgenic plants by targeting mannitol biosynthesis to chloroplasts. Plant Physiol. 113, 11771183.

Sleator, R. D., Gahan, C. G. M. and Hill, C. (2001) Mutations in the Listerial $p r o B$ gene leading to proline overproduction: effects on salt tolerance and murine infection. Appl. Environ. Microbiol. 67, 4560-4565.

Smith, C. J., Deuth, A. H. and Rushlow, K. E. (1984) Purification and characteristics of a $\gamma$-glutamyl kinase involved in Escherichia coli proline biosynthesis. J. Bacteriol. 157, 545551.

Su, J., Shen, Q., Ho, T. H. D. and Wu, R. (1998) Dehydrationstress regulated transgene expression in stably transformed rice plants. Plant Physiol. 117, 913-922.

Tarczynski, M. C., Jensen, R. G. and Bohnert, J. H. (1993) Stress protection of transgenic tobacco by production of the osmolyte Mannitol. Science 259, 508-510.

Whatmore, A. M., Chudek, J. A. and Reed, R. H. (1990) The effects of osmotic upshock on the intracellular solute pools of Bacillus subtilis. J. Gene. Microbiol. 136, 2527-2535.

Wood, J. M., Bremer, E., Csonka, L. N., Karaemer, R., Poolman, B., Van der Heide, T. and Smith, L. T. (2001) Osmosensing and osmoregulatory compatible solute accumulation by bacteria. Com. Biochem. Physiol. Part A 130, 437-460.

Zhang, C. S., Lu, Q. and Verma, D. S. P. (1995) Removal of feedback inhibition of delta-1-pyrroline-5-carboxylate synthetase, a bifunctional enzyme catalyzing the first 2 steps of proline biosynthesis in plants. J. Biol. Chem. 270, 20491-20496. 